NOTA CIENTÍFICA

\title{
Primer registro de Gracilinanus agilis (Burmeister, 1854) (Mammalia: Didelphidae) para Loreto, Perú
}

\section{First record of Gracilinanus agilis (Burmeister, 1854) (Mammalia: Didelphidae) for Loreto, Peru}

\section{Liz Huamaní1, Richard Cadenillas ${ }^{1}$ y Víctor Pacheco ${ }^{1,2}$}

\begin{abstract}
1 Departamento de Mastozoología, Museo de Historia Natural, Universidad Nacional Mayor de San Marcos, Av. Arenales 1256, Lima-14, Perú. Email Liz Huamani: lizsel_3@yahoo.es

2 Facultad de Ciencias Biológicas, Universidad Nacional Mayor de San Marcos, Av. Venezuela s/n, Lima-1, Perú.
\end{abstract}

Presentado: $\quad$ 19/09/2009 Aceptado: $\quad 09 / 12 / 2009$

Publicado online: $\quad 12 / 01 / 2010$

\section{Resumen}

Se reporta el primer registro de Gracilinanus agilis (Mammalia: Didelphidae) para el departamento de Loreto, Perú; el cual representa el registro más septentrional conocido de la especie.

Palabras clave: Gracilinanus agilis. Distribución. Selva baja.

\section{Abstract}

We report the first record of Gracilinanus agilis (Mammalia: Didelphidae) for the department of Loreto, Peru. This represents the northermost record of the species.

Keywords: Gracilinanus agilis. Distribution. Amazon rainforest.
En el departamento de Loreto, provincia de Maynas, distrito de Alto Nanay, Zona Reservada Pucacuro (2²9'35,88'S; 74059'0,6”W; 177 m), se colectó un ejemplar referible a Gracilinanus agilis en lo que constituye el primer registro del didélfido para el departamento de Loreto. Este espécimen (MUSM 24430) es una hembra juvenil de edad: clase 3 según Tribe (1990), colectado el 26 de octubre del año 2008 por Liz Huamaní (LHL 215), preparado como piel, cráneo y carcasa; y depositado en el Departamento de Mastozoología del Museo de Historia Natural de la Universidad Nacional Mayor de San Marcos.

El espécimen fue identificado como Gracilinanus por la presencia de los forámenes maxilares, maxilopalatinos y palatinos en el paladar óseo (Voss y Jansa 2003); ausencia de procesos supraor-

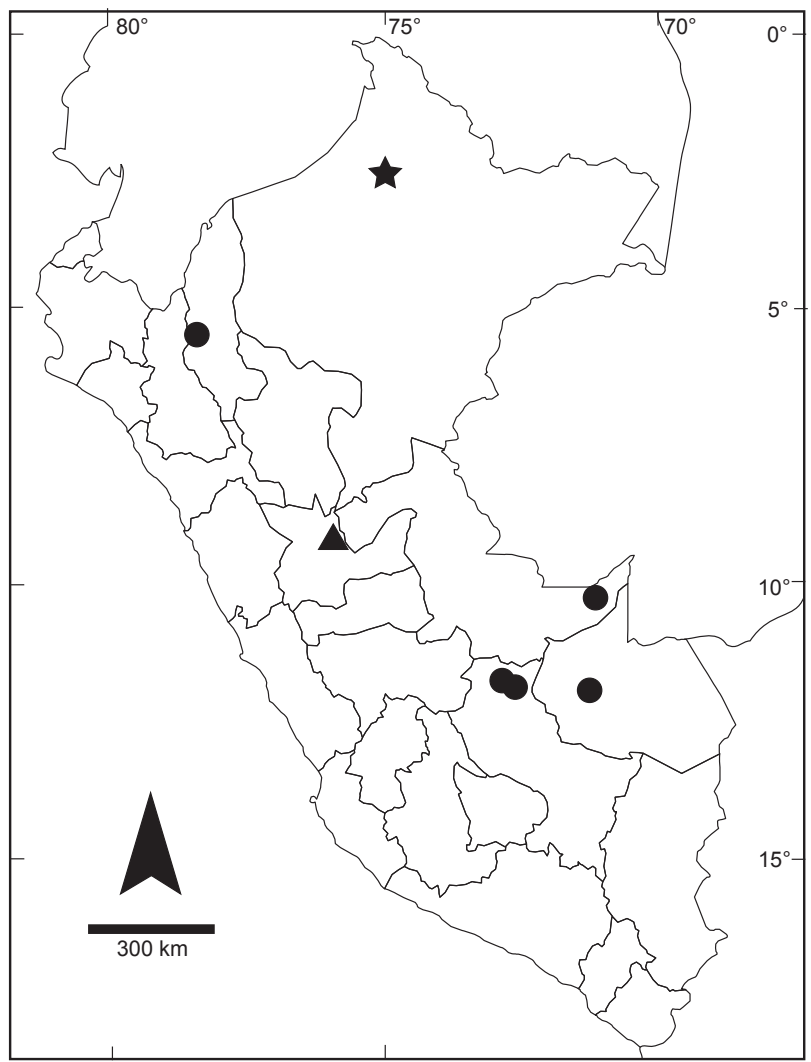

Figura 1. Registros de Gracilinanus agilis en el Perú: Nuevo registro (estrella negra), localidad tipo de G. agilis peruana (triángulo negro). Otros registros previos (círculo negro). bitales desarrollados; proceso anteromedial del ala timpánica del aliesfenoides cubriendo la vena carótida (Gardner y Creighton 1989); caninos (superiores e inferiores) con cúspide posterior accesoria (Voss et at. 2001) ; y relación del tamaño de la cola al cuerpo de 1,56 (Voss et al. 2005).. Este juvenil presenta el dpm3 molariforme tribosfénico, donde se distingue el talónido y un trigónido incompleto; mientras que en el DPM3 se distingue el protocono, paracono y metacono (Voss et at. 2001).

Además de estas características, el espécimen presenta el anillo ocular bien marcado alrededor del ojo y extendido hacia la nariz, el pelaje dorsal corto, marrón claro y la coloración del pelaje ventral blanquecina, que identifican a $G$. agilis y la diferencian de $G$. aceramarcae (Tate 1931). Según Creighton y Gardner (2008), este último taxón presenta el pelaje dorsal largo y la base del pelaje ventral de coloración gris. Nuestro espécimen presenta además medidas externas y craneales próximas al holotipo subadulto de Tate (1933), y más pequeńas que los especímenes adultos examinados de $G$. agilis (Tabla 1).

Según Tate (1933) nuestro espécimen correspondería a la subespecie peruana (Tate 1931). Sin embargo, seguimos a Creighton y Gardner (2008) quienes consideran a G. agilis como una especie monotípica en necesidad de revisión sistemática.

Según Creighton y Gardner (2008), G. agilis se encuentra presente en Brasil, Bolivia, Paraguay, Uruguay y cerca de Argentina, además de Perú, en contraste a Costa et al. (2003) que no incluyen la especie para Perú. Los autores no incluyen el registro de G. agilis de Colombia mencionado por Hershkovitz (1992) y Brown (2004), basados en un ejemplar de Unguia, debido a que dudan de la determinación de este espécimen. Además, Creighton y Gardner (2008) y Voss y Jansa (2009) mencionan que el registro de Amazonas, Bellavista, basado en tres pieles sin cráneos (Tate 1933), podría también no corresponder a la especie. Nuestro registro en la Zona Reservada Pucacuro, en comparación con los registros publicados de la especie (Creighton y Gardner 2008: Mapa 17) es el primer espécimen confirmado para Loreto, el primero al norte del Río Amazonas y el más septentrional conocido de la especie.

En Perú, la especie ha sido registrada también en: Madre de Dios, río Manu, Parque Nacional del Manu, puesto de vigilancia de Pakitza, 11056’47,04”S; 71¹6'59,88”W; 350 m 
Tabla 1. Medidas corporales y craneales de Gracilinanus agilis de Perú presentes en la colección mastozoológica del Museo de Historia Natural (MUSM). *medidas tomadas del holotipo de G. a. peruana en Tate (1933).

\begin{tabular}{|c|c|c|c|c|c|}
\hline \multirow{2}{*}{ Medidas } & \multicolumn{5}{|c|}{ Especímenes examinados } \\
\hline & MUSM 24430 & MUSM 8922 & MUSM 14085 & MUSM 14086 & ${ }^{*}$ BM 27.11.1.268 \\
\hline Sexo & 우 & 우 & q & 우 & $\hat{o}$ \\
\hline Edad & Juvenil & Adulto & Adulto & Adulto & Subadulto \\
\hline Longitud cabeza y cuerpo & 77 & 86 & 132 & 93 & 91 \\
\hline Longitud de la cola & 120 & 139 & 153 & 124 & 136 \\
\hline Longitud de la pata & 12 & 13 & 19 & 15 & 16 \\
\hline Longitud de la oreja & 14 & 15 & 23 & 15 & 18 \\
\hline Longitud máxima craneal & 21,06 & 25,27 & 35,61 & 25,93 & 26,3 \\
\hline Ancho cigomático & 11,64 & 14,29 & 17,75 & 14,6 & 15 \\
\hline Constricción interorbital & 4,05 & 4,45 & 6,06 & 6,67 & - \\
\hline Ancho postorbital & 4,68 & 5,45 & 6,5 & 4,61 & 6,2 \\
\hline Ancho de la caja craneal & 9,81 & 10,95 & 13,37 & 10,82 & 10,8 \\
\hline Longitud nasal & 7,93 & 9,4 & 16,35 & 10,07 & 9,6 \\
\hline Longitud I-M5 & 10,4 & 12,33 & 17,23 & 12,9 & 11,1 \\
\hline Longitud M2-5 & 3,9 & 4,98 & 6,42 & 5,23 & 5,9 \\
\hline Peso & 6 & 15 & 35 & - & - \\
\hline
\end{tabular}

(MUSM 8922, Pacheco et al. 1993); Cusco, La Convención, Echarate, Camisea, San Martín, 11046'59,88”S; 72049’W; 475 m (MUSM 14086, Solari et al 1999); Cusco, La Convención, Echarate, Camisea, Pagoreni, 11042'22”S; 72054'9”W; $450 \mathrm{~m}$ (MUSM 14085, Solari et al. 1999); Huánuco, Tingo María, río Huallaga, 98'S; 7557’W; 610 m (BM 27.11.1.268, Tate 1931); Amazonas, Bagua, Bellavista, valle seco del Marañón, 5²4’30”S; 78²6’24”W; 517 m (Tate 1933); y Ucayali, Purus, Balta, comunidad Cashinahua, 1008'S; 71¹3'W; $300 \mathrm{~m}$ (Voss y Emmons 1996). El rango de elevación de la especie en Perú es de 177 a 610 m, siendo nuestro espécimen el registro más bajo conocido de la especie en Perú; y confirmando su preferencia por el hábitat de selva baja tropical (Fig. 1).

En la Zona Reservada Pucacuro, G. agilis es simpátrica con ocho especies de marsupiales: Caluromys lanatus, Didelphis marsupialis, Marmosops impavidus, M. noctivagus, M. bishopi, Metachirus nudicaudatus, Marmosa (Micoureus) demerarae y Marmosa (Micoureus) regina (nomenclatura sensu Voss y Jansa 2009).

Nuestro espécimen fue colectado a mano fácilmente de un árbol a $2 \mathrm{~m}$ de altura, en horas de la noche, probablemente cegada por las linternas.

Agradecemos al Biólogo Raúl Navarro por su colaboración en la captura del espécimen, asimismo a las Biólogas Elena Vivar y Edith Salas por la ayuda en la identificación de la especie.

\section{Literatura citada}

Brown B. 2004. Atlas of New World marsupials. Fieldiana: Zoology, new series 102: 1-308.

Creighton G. \& A Gardner. 2008 (2007). Genus Gracilinanus Gardner y Creighton, 1989. Pp 43-50, en: Mammals of South America, Vol. 1. Marsupials, xenarthrans, shrews, and bats (AL Gardner, ed.). The University of Chicago Press. Chicago and London.

Costa, L.P., Y.L.R. Leite, and J.L. Patton. 2003. Phylogeography and systematic notes on two species of gracile mouse opossums, genus Gracilinanus (Marsupialia, Didelphidae) from Brazil. Proceedings of the Biological Society of Washington 116(2): 275-292.

Gardner A.L. \& G.K Creighton. 1989. A new generic name for Tate's microtarsus group of South American mouse opossums (Marsupialia: Didelphidae). Proceedings Biological Society of Washington 102: 3-7.
Hershkovitz P. 1992. The South American mouse opossums, Genus Gracilinanus Gardner and Creighton, 1989 (Marmosidae, Marsupialia): A taxonomic review with notes on general morphology and relationships. Fieldiana: Zoology, new series 70: $1-56$.

Pacheco V., B.D. Patterson, J.L. Patton, et al. 1993. List of mammal species known to occur in Manu Biosphere Reserve, Peru. Publicaciones del Museo de Historia Natural, Universidad Nacional Mayor de San Marcos (A) 44: 1-12.

Solari S., E. Vivar, J.J. Rodríguez, P.M. Velazco, et al. 1999. Small mammals: Biodiversity assessment at the Pagoreni well site. Pp 137-150, en: Biodiversity assessment and monitoring of the lower Urubamba region, Peru: Pagoreni well site assessment and training (A Alonso y F Dallmeier, eds.). Smithsonian Institution/Monitoring and Assessment of Biodiversity Program (SI/MAB) Serie 3. Washington, D.C.

Tate G.H.H. 1931. Brief diagnoses of twenty-six apparently new forms of Marmosa (Marsupialia) from South America. American Museum Novitates 493: 1-14.

Tate G. H.H. 1933. A Systematic Revision of the Marsupial Genus Marmosa. Bulletin of the American Museum of Natural History 66: 1-250.

Tribe C.J. 1990. Dental age classes in Marmosa incana and other didelphoids. Journal of Mammalogy 71(4): 566-569.

Voss R.S. \& L.H Emmons. 1996. Mammalian diversity in Neotropical lowland rainforests: A preliminary assessment. Bulletin of the American Museum of Natural History 230: 1-115.

Voss R.S. \& S.A. Jansa. 2003. Phylogenetic studies on didelphid marsupials II. Nonmolecular data and new IRBP sequenes: separate and combined analyses of didelphine relationships with denser taxon sampling. Bulletin of the American Museum of Natural History 276: 1-82.

Voss R.S. \& S.A. Jansa. 2009. Phylogenetic relationships and classification of didelphid marsupials, an extant radiation of New World metatherian mammals. Bulletin of the American Museum of Natural History 322: 1-117.

Voss R.S., D.P. Lunde \& N.B. Simmons. 2001. The mammals of Paracou, French Guiana: A neotropical lowland rainforest fauna. Part 2. Nonvolant species. Bulletin of the American Museum of Natural History 263: 1- 236.

Voss, R.S., D.P. Lunde, and S.A. Jansa. 2005. On the contents of Gracilinanus Gardner and Creighton, 1989, with the description of a previously unrecognized clade of small didelphid marsupials. American Museum Novitates 3482: $1-34$. 